INDUSTRIAL ASSESSMENT CENTERS

A PROGRAM OF DIRECT ASSISTANCE

FOR

$\begin{array}{cc}\text { SMALL AND MEDIUM-SIZE MANUFACTURERS } & \text { RECEIVED } \\ \text { JUN } 221998 \\ \text { Western Region } & \text { OST I } \\ \text { Quarterly Progress Report-March, 1998 }\end{array}$

Prepared for:

U. S. Department of Energy Office of Industrial Technologies
Agreement No. DE-FC01-92CE/40961 - T22
For additional information contact:

\author{
Gwen P'. Looby \\ Industrial Technology and \\ Energy Management \\ University City Science Center \\ (215) 387-1535, ext. 225
}

Date prepared: 17 June 1998

\title{
Summary of Activities
}

During the quarter ending March 31, 1998, the IACs in the Western Region issued assessment reports to 16 clients for the 1996-97 program period.

The attached summary, Table 1 , shows the aggregate numbers of industrial assessments performed by, reports received from, critiques completed and returned to, and implementation reports completed by each of the Western Region IACs under the 1995-96 program period through the quarter ending March 31, 1998. Table 2 shows the numbers of industrial assessments performed by, reports received from, critiques completed and returned to, and implementation reports completed by each of the Western Region IACs under the 1996-97 program period.

\section{Progress on Energy Audits, Industrial Assessments, and Implementation Reports}

Three hundred fifty-five industrial assessments have been completed for the 1996-97 program period and all of those reports have been received. All reports have been completed for the 1995-96 program period.

The IACs have collected most of the implementation data for the 1995-96 program period and have begun collecting the data for the 1996-97 program period.

\section{Other Activities}

Material was prepared for the IAC annual report for FY 96. 


\section{DISCLAMMER}

This report was prepared as an account of work sponsored by an agency of the United States Government. Neither the United States Government nor any agency thereof, nor any of their employees, makes any warranty, express or implied, or assumes any legal liability or responsibility for the accuracy, completeness, or usefulness of any information, apparatus, product, or process disclosed, or represents that its use would not infringe privately owned rights. Reference herein to any specific commercial product, process, or service by trade name, trademark, manufacturer, or otherwise does not necessarily constitute or imply its endorsement, recommendation, or favoring by the United States Government or any agency thereof. The views and opinions of authors expressed herein do not necessarily state or reflect those of the United States Government or any agency thereof. 


\section{DISCLAIMER}

Portions of this document may be illegible electronic image products. Images are produced from the best available original document. 


\begin{tabular}{|c|c|c|c|c|}
\hline \multicolumn{5}{|c|}{$\begin{array}{c}\text { Table 1 } \\
\text { Western Region } \\
\text { IAC Progress: Industrial Assessment Reports and Implementation Reports } \\
\text { Agreement No. DE-FC01-92CE-40961 } \\
\text { Program Period October 1, 1995, through September 30, } 1996 \\
\text { Results through March 31, } 1998\end{array}$} \\
\hline IAC & $\begin{array}{l}\text { Assessments } \\
\text { Completed }\end{array}$ & $\begin{array}{l}\text { Reports } \\
\text { Received }\end{array}$ & $\begin{array}{c}\text { Critiques } \\
\text { Completed and } \\
\text { Returned to IACs }\end{array}$ & $\begin{array}{l}\text { Implementation } \\
\text { Reports } \\
\text { Completed }\end{array}$ \\
\hline Arizona State University & 30 & 30 & 30 & 30 \\
\hline University of Arkansas-Little Rock & $29^{\circ}$ & 29 & 29 & 29 \\
\hline Bradley University & 30 & 30 & 30 & 30 \\
\hline Colorado State University & 30 & 30 & 30 & 30 \\
\hline lowa State University & 30 & 30 & 30 & 30 \\
\hline University of Kansas & 15 & 15 & 15 & 15 \\
\hline University of Missouri-Rolla & 30 & 30 & 30 & 30 \\
\hline University of Nevada-Reno & 30 & 30 & 30 & 30 \\
\hline Oklahoma State University & 30 . & 30 & 30 & 30 \\
\hline Oregon State University & $29^{*}$ & 29 & 29 & 29 \\
\hline San Diego State University & 30 & 30 & 30 & 30 \\
\hline San Francisco State University & 30 & 30 & 30 & 30 \\
\hline South Dakota State University & 30 & 30 & 30 & 30 \\
\hline Texas A\&M University-College Station & 30 & 30 & 30 & 30 \\
\hline Texas A\&M University-Kingsville & $29^{\circ}$ & 29 & 29 & 29 \\
\hline Total & 432 & 432 & 432 & 432 \\
\hline
\end{tabular}

- Each of these schools performed one assessment in a plant that was later determined to be outside of SICs 20-39. Those plants are not included in the totals. 


\begin{tabular}{|c|c|c|c|c|}
\hline \multicolumn{5}{|c|}{$\begin{array}{c}\text { Table 2 } \\
\text { Western Region } \\
\text { IAC Progress: Industrial Assessment Reports and Implementation Reports } \\
\text { Agreement No. DE-FC01-92CE-40961 } \\
\text { Program Period October 1, 1996, through September 30, } 1997 \\
\text { Results through March 31, 1998 }\end{array}$} \\
\hline IAC & $\begin{array}{l}\text { Assessments } \\
\text { Completed }\end{array}$ & $\begin{array}{l}\text { Reports } \\
\text { Received }\end{array}$ & $\begin{array}{c}\text { Critiques } \\
\text { Completed and } \\
\text { Returned to IACs }\end{array}$ & $\begin{array}{c}\text { Implementation } \\
\text { Reports } \\
\text { Completed } \\
\end{array}$ \\
\hline Arizona State University & 20 & 20 & 20 & 12 \\
\hline University of Arkansas-Little Rock & 25 & 25 & 25 & 1 \\
\hline Bradley University & 25 & 25 & 25 & 0 \\
\hline Colorado State University & 25 & 25 & 25 & 8 \\
\hline lowa State University & 25 & 25 & 25 & 0 \\
\hline University of Kansas & 15 & 15 & 15 & 0 \\
\hline University of Missouri-Rolla & 25 & 25 & 25 & 0 \\
\hline University of Nevada-Reno & 20 & 20 & 20 & 0 \\
\hline Oklahoma State University & 25 & 25 & 25 & 7 \\
\hline Oregon State University & 25 & 25 & 25 & 0 \\
\hline San Diego State University & 25 & 25 & 25 & 0 \\
\hline San Francisco State University & 25 & 25 & 25 & 0 \\
\hline South Dakota State University & 25 & 25 & 25 & 4 \\
\hline Texas A\&M University-College Station & 25 & 25 & 25 & 19 \\
\hline Texas A\&M University-Kingsville & 25 & 25 & 25 & 0 \\
\hline Total & 355 & 355 & 355 & 51 \\
\hline
\end{tabular}

